\title{
INTEGRAZIONE DI DIGESTATO CHIARIFICATO COME FONTE DI AZOTO PER LA COLTIVAZIONE DI MICROALGHE
}

\author{
ALESSANDRO ALGERI (*) \\ Nota presentata dal m.e. Stefano Maiorana \\ (Adunanza del 26 novembre 2015)
}

SUNTO. - Una possibile alternativa ai fertilizzanti classici per l'alimentazione di microalghe di acqua salata risulta essere la frazione liquida del digestato proveniente da impianti di digestione anaerobica. L'inclusione di questo nutriente tal quale determina tuttavia problematiche di crescita nella coltivazione dovute all'elevata torbidità. È stato quindi necessario progettare e sperimentare un processo di chiarificazione sostenibile per eliminare gran parte dei solidi sospesi presenti. Tramite l'impiego del mezzo di coltivazione proveniente dalla fase di filtrazione dell'alga è possibile flocculare termicamente i solidi di diversa dimensione presenti nel digestato, rendendolo meno torbido. L'impiego di questa soluzione in coltivazioni di Nannochloropsis di diversa scala, mostra buoni ratei di crescita e nessuna inibizione percettibile dovuta all'inclusione di digestato.

$* * *$

AвSTRACT. - A possible alternative to the common fertilizer for feeding microalgae is the liquid fraction of digestate coming from the plants of the anaerobic digestion plants. However, the inclusion of this nutrient as such leads to problems in the growth in culture due to the high turbidity. Therefore, it has been necessary to plan and test a process of sustainable clarification in order to remove most of the suspended solids that are present. By using the culture medium coming from the filtration phase of the alga, it is possible to thermally flocculate the solids of different size present in the digestate, thus making it less turbid. Testing the use of this solution in cultures at Nannochloropsis of different scale, it was observed that they have good growth rates and no perceptible inhibition due to the inclusion of the digestate.

(*) Università di Modena e Reggio Emilia, Italy.

E-mail: alessandro.algeri@gmail.com 


\section{INTRODUZIONE}

Il volume d'impiego di estratti microalgali per la produzione di principi attivi ad alto valore commerciale è fortemente influenzato dal costo di produzione della biomassa di partenza. Questo viene determinato dai nutrienti utilizzati che in particolare risultano essere anidride carbonica e fertilizzanti di diverso genere.

Per abbattere questa voce di costi è possibile impiegare sostanze nutritive di recupero che costituiscano fonti alternative di azoto, fosforo, carbonio e micronutrienti.

Un sottoprodotto presente in elevata quantità, di difficile smaltimento e la cui efficacia come nutriente per microalghe è già stata studiata [1][2], risulta essere la fase liquida del digestato proveniente da impianti di produzione di biogas.

Questa materia risulta includere tenori di azoto totale piuttosto elevati, tracce di fosforo sotto forma di fosfato solubile e micronutrienti utili per l'alga. La composizione e le concentrazioni di questi nutrienti risultano tuttavia variabili in funzione dell'alimentazione dell'impianto di digestione anaerobica.

La principale criticità del sottoprodotto preso in esame risulta essere la presenza di particolato in sospensione con granulometria variabile da $0,2 \mathrm{~mm}$ a $3 \mu \mathrm{m}$. Questo determina un'alta torbidità del liquido, anche se diluito, ed una conseguente schermatura della luce nella coltivazione.

Per rendere utilizzabile il digestato è quindi indispensabile procedere con una chiarificazione che può avvenire con metodi fisici oppure chimici.

\section{ANALISI DEL REFLUO LIQUIDO PROVENIENTE DALL'IMPIANTO DI DIGESTIONE CON DETERMINAZIONE DEI LIVELLI DI FOSFORO, AZOTO, SOLIDI SOSPESI E METALLI PESANTI}

Per la sperimentazione svolta è stato utilizzato digestato liquido proveniente dall'impianto di digestione anaerobica di proprietà di Mantovagricoltura snc sito in Via Malpensa 5/a, Rodigo MN. L'impianto è alimentato tramite scarti provenienti da raccolta differenziata urbana, industriale e fonti amidacee vegetali.

Il digestato ottenuto viene preventivamente separato dalla fase solida grossolana mediante un filtro. 
Tab. 1 e Tab. 2 mostrano le analisi svolte sulla matrice liquida per la determinazione dei macronutrienti e dei principali metalli pesanti presenti.

Tab. 1 - Analisi macro-componenti digestato liquido.

\begin{tabular}{llll}
\hline Prova & Risultato & Unità di misura & Metodo \\
\hline Azoto totale & 0,66 & $\%$ & UNI EN 1550-2009 \\
\hline Calcio $(\mathrm{Ca})$ & 0,19 & $\%$ & Calcolo \\
\hline Fosforo $(\mathrm{P})$ & 549,1 & $\mathrm{mg} / \mathrm{Kg}$ & Calcolo \\
\hline Potassio $(\mathrm{K})$ & 530,0 & $\mathrm{mg} / \mathrm{Kg}$ & Calcolo \\
\hline Magnesio $(\mathrm{Mg})$ & 402,4 & $\mathrm{mg} / \mathrm{Kg}$ & Calcolo \\
\hline Boro $(\mathrm{B})$ & 1,08 & $\mathrm{mg} / \mathrm{Kg}$ & Calcolo \\
\hline Zinco $(\mathrm{Zn})$ & 5,66 & $\mathrm{mg} / \mathrm{Kg}$ & Calcolo \\
\hline Cloruri $(\mathrm{Cl})$ & 2410,8 & $\mathrm{mg} / \mathrm{Kg}$ & UNI ISO 9297 \\
\hline
\end{tabular}

Tab. 2 - Metalli pesanti digestato liquido.

\begin{tabular}{llll}
\hline Prova & Risultato & Unità di misura & Metodo \\
\hline Cromo VI & $<0,1$ & $\mathrm{mg} / \mathrm{Kg}$ s.s. & EPA 3050B 1996 \\
\hline Cadmio $(\mathrm{Cd})$ & $<0,1$ & $\mathrm{mg} / \mathrm{Kg}$ s.s. & CNR IRSA 16 Q 64 Vol. 31986 \\
\hline Piombo $(\mathrm{Pb})$ & 6,8 & $\mathrm{mg} / \mathrm{Kg}$ s.s. & EPA 3050B 1996 \\
\hline Rame $(\mathrm{Cu})$ & 56,1 & $\mathrm{mg} / \mathrm{Kg}$ s.s. & EPA 3050B 1996 \\
\hline Zinco $(\mathrm{Zn})$ & 277,9 & $\mathrm{mg} / \mathrm{Kg}$ s.s. & EPA 3050B 1996 \\
\hline Mercurio $(\mathrm{Hg})$ & $<0,2$ & $\mathrm{mg} / \mathrm{Kg}$ s.s. & MI-A016 Rev. 00/2013 \\
\hline Nichel $(\mathrm{Ni})$ & 13,4 & $\mathrm{mg} / \mathrm{Kg}$ s.s. & EPA 3050B 1996 \\
\hline
\end{tabular}

\subsection{Prove di diversi tipi di pretrattamenti per eliminare $i$ solidi dal digestato}

Tutti gli esperimenti sono stati compiuti in laboratorio eseguendo le prove in doppio o in triplo nel caso di risultati discordanti.

I solidi sospesi presenti nella fase liquida del digestato sono principalmente cellule batteriche morte, biomassa non digerita o digerita parzialmente tra cui lignina e cellulosa, metalli e sali organici e inorganici che spesso creano dispersioni.

La separazione per sedimentazione risulta fondamentale per eliminare il particolato non sospeso ma poco efficiente per le particelle di più piccole dimensioni. 


\subsection{Trattamento a caldo con soluzione ad alta forza ionica}

Il primo metodo di flocculazione per eliminare il particolato presente in soluzione consiste nell'aumentare la forza ionica della soluzione tramite $\mathrm{NaCl}$, portare quest'ultima a $\mathrm{pH}$ acido (2-3) tramite un acido inorganico e successivamente riscaldare fino a $60^{\circ} \mathrm{C}$. Una volta fatto precipitare il solido centrifugare per dividere le due fasi ed ottenere un surnatante limpido.

Il metodo è stato implementato durante la sperimentazione tramite le seguenti prove:

Partendo da campioni di $10 \mathrm{ml}$ di digestato essi sono stati trattati con $5 \mathrm{~g}$ di $\mathrm{NaCl}$ in eccesso e successivamente portati a $\mathrm{pH}$ differenti (da 1 a 10). I campioni basificati non mostravano coagulazione di particelle e centrifugandoli a $3000 \mathrm{rpm}$ per 2 minuti si sono ottenuti risultati assimilabili al digestato tal quale. Nei campioni a $\mathrm{pH}$ acido si è notato invece un progressivo precipitare di agglomerati di solido e centrifugando nelle stesse condizioni si è ottenuta una soluzione limpida. In particolare sono stati presi in considerazione i campioni a $\mathrm{pH} 1-3$ per i successivi esperimenti.

Per determinare la quantità ottimale di $\mathrm{NaCl}$ necessario per ottenere la precipitazione del solido sono stati testati campioni da $100 \mathrm{ml} \mathrm{di}$ digestato a cui sono stati aggiunti 1,2,3 e 5 grammi di cloruro di sodio e successivamente acidificati a $\mathrm{pH} 1$ tramite un acido inorganico.

Già dal campione contenente $2 \mathrm{~g}$ di $\mathrm{NaCl}$ il digestato risulta ben flocculato, si è quindi proceduto mantenendo questa concentrazione.

Le prove per determinare la temperatura a cui far avvenire la flocculazione hanno previsto campioni da $100 \mathrm{ml}$ contenenti $2 \mathrm{~g}$ di $\mathrm{NaCl}$ e acidificati a $\mathrm{pH} 1$. Le flocculazioni sono state condotte a temperatura ambiente, a $40^{\circ} \mathrm{C}$, a $60^{\circ} \mathrm{C}$, a $80^{\circ} \mathrm{C}$, e a $110^{\circ} \mathrm{C}$. Le flocculazioni dalle quali si sono ottenuti risultati migliori sia come tempo di flocculazione che come efficienza sono le due a 110 e $80^{\circ} \mathrm{C}$. Tuttavia un pretrattamento del genere implica un riscaldamento elevato e un conseguente consumo termico, si è optato quindi per un trattamento a $60^{\circ} \mathrm{C}$ come buon compromesso tra efficienza ed economicità.

\subsection{Trattamento a caldo con soluzione a media forza ionica}

Terminate le prove sull'ottimizzazione del trattamento con $\mathrm{NaCl}$ si è deciso di esplorare una variante per le colture algali di acqua salata. 
Per questo tipo di alghe è inutile aggiungere $\mathrm{NaCl}$ solido poiché il mezzo di coltivazione ne contiene già $25 \mathrm{~g}$ per litro.

Si è proceduto allora a sperimentare la flocculazione con diverse proporzioni tra acqua di mare artificiale e digestato, acidificando e scaldando come sopra descritto.

Rese accettabili sono state ottenute con due parti di digestato e una parte di acqua di mare. Per questo tipo di pretrattamento, dove $\mathrm{i}$ solidi disciolti vengono diluiti, è necessario operare ad una temperatura di almeno $80^{\circ} \mathrm{C}$. Riuscire a flocculare senza $\mathrm{NaCl}$ solido permette di integrare meglio la fase nel ciclo produttivo delle alghe. In particolare sfruttando l'acqua priva di alghe in uscita dal raccoglitore che, prima di essere recuperata, viene utilizzata per il pretrattamento del digestato.

\subsection{Neutralizzazione}

I reattori utilizzati per la coltivazione di microalghe solitamente operano a $\mathrm{pH}$ compresi tra 6 e 10, è quindi necessario basificare la soluzione surnatante da cui sono stati eliminati la maggior parte dei solidi sospesi.

Portando il $\mathrm{pH}$ da acido a neutro si è assistito tuttavia alla formazione di altro precipitato di colore nerastro, superato il valore indicativo di 5.5 gradi di $\mathrm{pH}$. Eliminando il solido la soluzione risulta molto più limpida rispetto alla precedente a $\mathrm{pH}$ acido.

\subsection{Trattamento tramite un flocculante inorganico}

Un metodo classico per la "chiarificazione" del digestato dai solidi sospesi consiste nell'impiego di un flocculante inorganico convenzionale: il cloruro ferrico. [3]

Questa sostanza permette sia di sfruttare l'effetto coagulante dei cloruri che quello dello ione ferro per ottenere una buona flocculazione a concentrazioni meno elevate.

Partendo da campioni di digestato da $100 \mathrm{ml}$ si sono aggiunti rispettivamente soluzioni di $\mathrm{FeCl}_{3}$ a $0,5 \% 1 \% 2 \%$ a campioni differenti e si è attesa per alcuni minuti la flocculazione agitando.

Tutti i campioni sono stati successivamente centrifugati ed i surnatanti ottenuti sono stati confrontati.

Tutte le concentrazioni di $\mathrm{FeCl}_{3}$ testate hanno prodotto un surnatante limpido. Le soluzioni derivanti da questo pretrattamento necessitano di essere neutralizzate ma non riseparate. 
I prodotti ottenuti dopo la flocculazione con la soluzione salina proveniente dalla raccolta dell'alga e con $\mathrm{FeCl}_{3}$ risultano essere comparabili sia per la torbidità che per concentrazione di azoto. Si è quindi optato per sfruttare il primo metodo per le prove svolte sulle coltivazioni.

\subsection{Analisi nutrienti e cloruri dopo la flocculazione}

Tab. 3 - Analisi macro-composizione digestato flocculato con acqua marina artificiale.

\begin{tabular}{llll}
\hline Prova & Risultato & Unità di misura & Metodo \\
\hline Azoto totale & 0,37 & $\%$ & UNI EN 1550-2009 \\
\hline Azoto ammoniacale & 3023 & $\mathrm{mg} / \mathrm{Kg}$ & MP 002 NH5+ 01/02 \\
\hline Fosforo $(\mathrm{P})$ & 21,4 & $\mathrm{mg} / \mathrm{Kg}$ & Calcolo \\
\hline Cloruri & 13323 & $\mathrm{mg} / \mathrm{Kg}$ & UNI ISO 9297 \\
\hline
\end{tabular}

Il notevole aumento dei cloruri era previsto date le aggiunte di acqua salata nel pretrattamento. La presenza pressoché quantitativa dei cloruri in soluzione permette di utilizzare il flocculato solido in agricoltura.

Come previsto il livello di fosforo cala drasticamente dopo il pretrattamento, è quindi necessario procedere ristabilendo il rapporto azoto fosforo a livelli ottimali (Tab. 3).

\section{INTEGRAZIONE DEL GENERE NANNOCHLOROPSIS CON I TIPI DI REFLUI PRETRATTATI A DIVERSE CONCENTRAZIONI}

Un'analisi iniziale delle caratteristiche di diversi generi di microalghe, accomunati da un possibile impiego per la produzione di principi attivi di interesse commerciale, è stata effettuata per determinare la miglior alternativa con la quale condurre la sperimentazione.

Si è tratto che Nannochloropsis fosse la migliore alternativa, vista la resistenza ad alti tenori di azoto e la buona integrazione con il digestato. [4][5][6] Il ceppo scelto di Nannochloropsis sp è stato coltivato in autotrofa con diverse concentrazioni di digestato trattato.

Le coltivazioni sotto riportate sono state sperimentate in doppio utilizzando, quando non precisato, lampade Philips DayLight 58W come fonte di illuminazione. Tutte le coltivazioni sono state condotte a temperatura ambiente durante il periodo estivo in laboratorio con temperature che variavano dai 27 ai $36^{\circ} \mathrm{C}$.

Le coltivazioni sono state preparate aggiungendo rispettivamente 
5, 10, 20,30 $\mathrm{ml}$ di digestato in diverse beute da $300 \mathrm{ml}$ e portandole tutte a $100 \mathrm{ml}$ tramite il mezzo di coltivazione $\mathrm{f} / 2$ [7]. Tutte le beute sono state poste in autoclave a due atmosfere per 20 minuti per eliminare eventuali tracce batteriche.

Ad ogni beuta è stato poi aggiunto un inoculo di Nannochloropsis per raggiungere una concentrazione finale di circa $0,1 \mathrm{~g} / \mathrm{L}$.

Il periodo di coltivazione previsto è stato di 30 giorni in modo da comprendere eventuali acclimatazioni e lag da parte dell'alga.

Le coltivazioni contenenti $30 \mathrm{ml}$ di digestato non hanno mostrato crescita e dopo 30 giorni non si è riscontrata la presenza di alga ancora viva in soluzione.

Le coltivazioni contenenti $20 \mathrm{ml}$ di digestato hanno presentato un rateo di crescita molto basso ed un lag iniziale di due settimane.

Le coltivazioni contenenti $10 \mathrm{ml}$ di digestato hanno presentato una lag di 5 giorni seguita da una crescita fino ad una concentrazione massima di $0,9 \mathrm{~g} / \mathrm{L}$.

Prove successive hanno dimostrato che procedendo gradualmente con l'aumento della concentrazione di digestato, partendo da una base al $10 \%$, può portare la coltivazione a mantenere un buon rateo di crescita fino ad una concentrazione di digestato al $20 \% \mathrm{v} / \mathrm{v}$.

La terza fase di prove riguardante il ceppo di Nannochloropsis, contenente una concentrazione di digestato al 10\%, ha previsto il confronto della crescita tra coltivazioni contenenti il digestato chiarificato e il mezzo oppure soltanto il mezzo. A concentrazioni comprese tra il 10 e il $20 \%$ di digestato si è potuto notare un aumento della crescita rispetto alle coltivazioni contenenti soltanto il mezzo.

\subsection{Scalaggio della coltivazione in reattore}

Per determinare la variazione della crescita dell'alga in condizioni di volumetrie maggiori si sono progettati e costruiti due fotobioreattori dalla capacità di 30 e $300 \mathrm{~L}$. Il primo ha permesso l'inoculo del ceppo nel secondo e il mantenimento di uno starter attivo.

Entrambi i fotobioreattori sono alimentati a luce LED fornita da diodi commerciali SMD 50:50 emittenti frequenze corrispondenti a luce bianca fredda e calda. [8][9]

La coltivazione in entrambi i fotobioreattori è stata condotta utilizzando mezzo di coltivazione $\mathrm{f} / 2$ [7] privo di azoto sia in forma nitrica che ammoniacale e contenente il $10 \%$ di digestato chiarificato. 
Le condizioni operative hanno previsto un periodo di luce e buio di 16 e 8 ore rispettivamente per entrambi i reattori.

I test sono stati svolti nella stagione estiva e hanno visto oscillazioni di temperature della coltivazione tra i 25 e i $31^{\circ} \mathrm{C}$.

I dati di crescita presi in esame sono stati ottenuti dopo 20 giorni dall'inoculo dell'alga nel reattore da 300 L ad una concentrazione iniziale di circa $0,1 \mathrm{~g} / \mathrm{L}$.

La concentrazione finale dell'alga è risultata essere di $1,1 \mathrm{~g} / \mathrm{L}$ nel reattore da 300L e di 1,2 g/L nel reattore da 30L, il che dimostra un buon rateo di crescita di Nannochloropsis sfruttando digestato chiarificato come fonte di azoto inorganico.

\section{CONCLUSIONI}

La chiarificazione della fase liquida del digestato tramite acqua marina proveniente dalla raccolta dell'alga risulta essere efficace e poco costosa, tenendo in considerazione la disponibilità di calore emesso dal cogeneratore e utilizzabile per il riscaldamento della miscela acqua/digestato. Il processo di separazione del flocculato può avvenire anche per semplice sedimentazione, non apportando ulteriori costi al processo.

Il digestato trattato in questo modo è risultato essere un'alternativa valida alle fonti classiche di azoto inorganico nella coltivazione di Nannochloropsis.

\section{BIBLIOGRAFIA}

Uggetti E., Sialve B., Latrille E., Steyer J.P., "Anaerobic digestate as substrate for microalgae culture: the role of ammonium concentration on the microalgae productivity.", Bioresource technology 152, (2014): 437-443.

Sepúlveda C., Acién F. G., Gómez C., Jiménez-Ruíz N., Riquelme C., Molina-Grima E. "Utilization of centrate for the production of the marine microalgae Nannochloropsis gaditana.”, Algal Research 9, (2015): 107-116.

Waeger F., Delhaye T., Fuchs W., "The use of ceramic microfiltration and ultrafiltration membranes for particle removal from anaerobic digester effluents." Separation and Purification Technology 73.2, (2010): 271-278.

Sheets J.P., Ge X., Park S.Y., Li Y., "Effect of outdoor conditions on Nannochloropsis salina cultivation in artificial seawater using nutrients from anaerobic digestion effluent." Bioresource technology 152, (2014): 154-161. 
Cai T., Park S.Y., Racharak R., Li Y., "Cultivation of Nannochloropsis salina using anaerobic digestion effluent as a nutrient source for biofuel production." Applied energy 108 (2013): 486-492.

Dong B., Ho N., Ogden K.L., Arnold R.G., "Cultivation of Nannochloropsis salina in municipal wastewater or digester centrate." Ecotoxicology and environmental safety 103, (2014): 45-53.

Guillard, Robert RL, and John H. Ryther, "Studies of marine planktonic diatoms: I. Cyclotella Nana Hustedt, and Detonula Confervacea (CLEVE) Gran." Canadian journal of microbiology 8.2, (1962): 229-239.

Kandilian R., Lee E., Pilon L., "Radiation and optical properties of Nannochloropsis oculata grown under different irradiances and spectra." Bioresource technology 137, (2013): 63-73.

Vadiveloo A., Moheimani N.R., Cosgrove J.J., Bahri P.A., Parlevliet D., "Effect of different light spectra on the growth and productivity of acclimated Nannochloropsis sp.(Eustigmatophyceae).” Algal Research 8, (2015): 121-127. 